\title{
Some two-dimensional extensions of Bougerol's identity in law for the exponential functional of linear Brownian motion
}

\author{
Jean Bertoin, Daniel Dufresne and Marc Yor
}

\begin{abstract}
We present a two-dimensional extension of an identity in distribution due to Bougerol [4] that involves the exponential functional of a linear Brownian motion. Even though this identity does not extend to the level of processes, we point out further striking relations in this direction.
\end{abstract}

\section{Introduction}

1.1. To a linear Brownian motion $\left(B_{s}, s \geq 0\right)$ starting from 0 , we associate the exponential functional

$$
A_{t}=\int_{0}^{t} \exp \left(2 B_{s}\right) d s, \quad t \geq 0 .
$$

The distribution of $A_{t}$ is made accessible thanks to Bougerol's identity in law:

$$
\text { for fixed } t, \quad \sinh \left(B_{t}\right) \stackrel{(\text { law })}{=} \beta\left(A_{t}\right),
$$

where $(\beta(u), u \geq 0)$ denotes a Brownian motion which is independent of $\left(B_{s}\right.$, $s \geq 0$ ), hence of $A_{t}$. Assuming (1.1), elementary computations yield the characterization of the law of $A_{t}$ as

$$
\mathbb{E}\left[\frac{1}{\sqrt{A_{t}}} \exp \left(-\frac{x^{2}}{2 A_{t}}\right)\right]=\frac{a^{\prime}(x)}{\sqrt{t}} \exp \left(-\frac{a^{2}(x)}{2 t}\right), \quad x \in \mathbb{R},
$$

where

$$
a(x)=\arg \sinh (x) \equiv \log \left(x+\sqrt{1+x^{2}}\right) \quad \text { and } \quad a^{\prime}(x)=\frac{1}{\sqrt{1+x^{2}}} .
$$

Mathematics Subject Classification (2010): Primary 60J65, 60J60; Secondary 60J55.

Keywords: Brownian motion, exponential functional, Bougerol's identity, local time, Bessel processes. 
For further reference, we note the simple, but useful, consequences of (1.2) that

$$
\mathbb{E}\left(\frac{1}{\sqrt{A_{t}}}\right)=\frac{1}{\sqrt{t}}
$$

and, differentiating both sides of (1.3) with respect to $t$,

$$
\mathbb{E}\left(\frac{\exp \left(B_{t}\right)}{A_{t}^{3 / 2}}\right) \stackrel{(*)}{=} \mathbb{E}\left(\frac{\exp \left(2 B_{t}\right)}{A_{t}^{3 / 2}}\right)=\frac{1}{t^{3 / 2}},
$$

where $(*)$ is obtained by time reversal of $\left(B_{s}, s \leq t\right)$ from time $t$.

1.2. It took some time, despite the original proof in [4], to understand simply the deeper reasons why (1.1) holds. In [1], one finds the following arguments, among which the (essential) time reversal one:

$$
\beta\left(A_{t}\right) \text { is distributed as } \int_{0}^{t} \exp \left(B_{s}\right) d \beta(s) \text { for fixed } t,
$$

and, by time reversal (at time $t$ ), this is also distributed as

$$
\exp \left(B_{t}\right) \int_{0}^{t} \exp \left(-B_{s}\right) d \beta(s)
$$

Now, it is easily shown, using Itô's formula, that the process in (1.5) is distributed as the process $\left(\sinh \left(B_{t}\right), t \geq 0\right)$.

1.3. In the present paper, we obtain an extension of (1.1), by considering the two-dimensional vector $\left(\sinh \left(B_{t}\right), \sinh \left(L_{t}\right)\right)$, where $\left(L_{t}, t \geq 0\right)$ denotes the local time at 0 of $B$. Our main result is:

Theorem 1.1. For fixed $t$, the three following two-dimensional random variables are identically distributed:

$$
\begin{aligned}
\left(\sinh \left(B_{t}\right), \sinh \left(L_{t}\right)\right) \stackrel{\stackrel{\text { law })}{=}}{=}\left(\beta\left(A_{t}\right), \exp \left(-B_{t}\right) \lambda\left(A_{t}\right)\right) \\
\stackrel{\text { (law) }}{=}\left(\exp \left(-B_{t}\right) \beta\left(A_{t}\right), \lambda\left(A_{t}\right)\right),
\end{aligned}
$$

where $(\beta(u), u \geq 0)$ is a one-dimensional Brownian motion, with local time at 0 , $(\lambda(u), u \geq 0)$, and $\beta$ is independent of $B$.

It may be interesting to observe that Tanaka's formula shows that the local time at level 0 and time $t$ of the process $\left(\sinh \left(B_{s}\right), s \geq 0\right)$ is simply $L_{t}$, whereas that of the process $\left(\exp \left(-B_{s}\right) \beta\left(A_{s}\right), s \geq 0\right)$ can be expressed as $\int_{0}^{t} \exp \left(-B_{s}\right) d \lambda\left(A_{s}\right)$. Hence we have also the identity in distribution between two-dimensional processes,

$$
\left(\sinh \left(B_{t}\right), L_{t}\right)_{t \geq 0} \stackrel{(\text { law })}{=}\left(\exp \left(-B_{t}\right) \beta\left(A_{t}\right), \int_{0}^{t} \exp \left(-B_{s}\right) d \lambda\left(A_{s}\right)\right)_{t \geq 0} .
$$

We stress that (1.6) cannot be extended to the level of processes; see Section 2.2. Hence the two identities in distribution (1.6) and (1.7) differ profoundly. 
Theorem 1.1 is proved in Section 3. In Section 2, we discuss a number of consequences of and statements equivalent to Theorem 1.1. For instance, the well-known equivalence in law, due to Paul Lévy, between the processes $\left(\left(\bar{B}_{t}-B_{t}, \bar{B}_{t}\right), t \geq 0\right)$ and $\left(\left(\left|B_{t}\right|, L_{t}\right), t \geq 0\right)$ allows to present a version involving the supremum $\bar{B}_{t}=$ $\sup _{s \leq t} B_{s}$ instead of the local time version of Theorem 1.1.

Apart from this, Section 2 consists in the statements and discussions of four other theorems. Roughly speaking, these theorems were motivated by our desire to understand whether in (1.6) the two extreme identities hold for processes. This question has now been solved in the negative (see [3]), but nonetheless there are some rather remarkable identities between jump intensity measures, which are described in Theorems 2.3-2.6, and which made us believe for some time in a 2-dimensional process identity extending (1.6). We let the reader discover the precise statements of these theorems in Section 2; their proofs are found in Section 4.

1.4. Before we enter into the details of the proofs of our theorems, we make some remarks explaining how our understanding of Bougerol's identity (1.1) has developed: in [19], a Mellin transform proof was given, based on the identity in law (3.6) below. Later, in [1], a time-reversal argument and stochastic calculus proof of (1.1) were found. We feel that, at the moment, our understanding of Theorem 1.1 lies at the level of [19], and that it should be possible to develop some kind of understanding similar to that in [1]. However, such a proof eludes us for now; it is not clear that a time-reversal argument is what is missing. Nevertheless, we present some further extensions of Bougerol's identity different from the ones found in the volume [20], which, hopefully will lead us in the future to a better understanding of Theorem 1.1. We also refer to [17] for a recent survey of this topic.

\section{Discussion of and some theorems closely related to Theo- rem 1.1}

2.1. First we note that we may rewrite the identity in law (1.6) in the seemingly slightly weaker form

$$
\begin{aligned}
\left(\sinh \left(\left|B_{t}\right|\right), \sinh \left(L_{t}\right)\right) \stackrel{\stackrel{\text { law })}{=}}{=}\left(|\beta|\left(A_{t}\right), \exp \left(-B_{t}\right) \lambda\left(A_{t}\right)\right) \\
\stackrel{\text { (law) }}{=}\left(\exp \left(-B_{t}\right)|\beta|\left(A_{t}\right), \lambda\left(A_{t}\right)\right) .
\end{aligned}
$$

This supposes no loss of generality, since the expressions on the left-hand side (without absolute values) of (1.6) only differ from the expressions with absolute values in (2.1) by multiplication by a symmetric Bernoulli variable, independent of the remaining quantities.

Secondly, it is well known that the law of the two-dimensional vector $\left(\left|B_{t}\right|, L_{t}\right)$ is symmetric. More precisely, it is given by

$$
\mathbb{P}\left(\left|B_{t}\right| \in d x, L_{t} \in d \ell\right)=\frac{2(x+\ell)}{\sqrt{2 \pi t^{3}}} \exp \left(-\frac{(x+\ell)^{2}}{2 t}\right) d x d \ell, \quad x \geq 0, \ell \geq 0,
$$


as can be checked using Lévy's identity (Theorem VI.2.3 in [15], p. 240) and the reflection principle (Exercise 3.14 in [15], p. 110, or Proposition 2.8.1 in [10], p. 95). Hence, the common law of (2.1) is also symmetric.

2.2. We now consider the second elements of the first and third vectors in (1.6) (or (2.1)), and we deduce from there

$$
\sinh \left(L_{t}\right) \stackrel{\text { law })}{=} \lambda\left(A_{t}\right) .
$$

Now, unlike for Bougerol's identity (1.1), for which the possibility of an identity in law between processes is immediately ruled out, since the left-hand side of (1.1) is not a martingale, whereas the right-hand side is, when one considers (2.3) it seems reasonable to wonder whether this identity might be valid at the level of the two increasing processes involved. However, the recent results in [3] rule out this possibility. In fact, it is this uncertainty which prevented us from publishing the earlier version of this paper [9].

We point out also that for each fixed $t \geq 0$, we deduce from (1.7) the rather puzzling identity in law

$$
\lambda\left(A_{t}\right) \stackrel{(\text { law })}{=} \sinh \left(\int_{0}^{t} \exp \left(-B_{s}\right) d \lambda\left(A_{s}\right)\right),
$$

which complements (2.3).

2.3. Reformulation in terms of Brownian suprema. A celebrated identity in distribution due to Paul Lévy states that

$$
\left(\left(\bar{B}_{t}-B_{t}, \bar{B}_{t}\right), t \geq 0\right) \stackrel{(\text { law })}{=}\left(\left(\left|B_{t}\right|, L_{t}\right), t \geq 0\right)
$$

where $\bar{B}_{t}=\sup _{s<t} B_{s}$ denotes the supremum of the Brownian trajectory up to time $t$. This enables us to reformulate (2.1) in the form

$$
\begin{aligned}
\left(\sinh \left(\bar{B}_{t}-B_{t}\right), \sinh \left(\bar{B}_{t}\right)\right) \stackrel{\text { (law) }}{=}\left((\bar{\beta}-\beta)\left(A_{t}\right), \exp \left(-B_{t}\right) \bar{\beta}\left(A_{t}\right)\right) \\
\stackrel{\text { (law) }}{=}\left(\exp \left(-B_{t}\right)(\bar{\beta}-\beta)\left(A_{t}\right), \bar{\beta}\left(A_{t}\right)\right),
\end{aligned}
$$

with $\bar{\beta}(t)=\sup _{s \leq t} \beta(s)$. We leave to the interested reader further alternative reformulations of this identity in the same vein.

2.4. A partial interpretation in terms of the Bessel clock. We now discuss Bougerol's identity (1.1) in terms of a two-dimensional Bessel process. Specifically, let $\left(R_{h}, h \geq 0\right)$ denote 2 -dimensional Bessel process starting from 1 , and let

$$
H_{u}=\int_{0}^{u} \frac{d h}{R_{h}^{2}}, \quad u \geq 0,
$$

be the clock associated with $R$. The well-known skew-product decomposition of planar Brownian motion ([12], p. 270; [13]; [14]) shows that the clock $H$ can be viewed as the inverse of the exponential Brownian functional $A$; consequently, considering the inverses of the increasing processes involved in (2.3), we obtain the following: 
Corollary 2.1. Let $\left(\sigma_{t}, t \geq 0\right)$ denote a stable (1/2) subordinator. Precisely,

$$
\sigma_{t}:=\inf \left\{u: \lambda_{u} \geq t\right\}, \quad t \geq 0,
$$

independent of $\left(R_{h}, h \geq 0\right)$. Then, for fixed $s$, one has

$$
H_{\sigma_{s}} \stackrel{(\text { law })}{=} \sigma_{a(s)}
$$

where $a(s) \equiv \arg \sinh (s)$.

It is interesting to compare Corollary 2.1 with the following consequence of (1.7):

Corollary 2.2. There is the identity between processes

$$
\left(\sigma_{t}\right)_{t \geq 0} \stackrel{(\text { law })}{=}\left(H_{\sigma_{\eta(t)}}\right)_{t \geq 0},
$$

where $\sigma$ is as in Corollary 2.1 and $\eta:[0, \infty) \rightarrow[0, \infty)$ is the inverse bijection of the continuous strictly increasing process

$$
s \mapsto \int_{0}^{s} \frac{d u}{R_{\sigma_{u}}} .
$$

Proof. Indeed, in terms of the Bessel clock, (1.7) yields

$$
\left(L_{t}\right)_{t \geq 0} \stackrel{(\text { law })}{=}\left(\int_{0}^{A_{t}} \frac{d \lambda(u)}{R_{u}}\right)_{t \geq 0} .
$$

Our statement now follows from the easy fact that the process $\left(\sigma_{\eta(t)}, t \geq 0\right)$ is the right inverse of the continuous increasing process $s \mapsto \int_{0}^{s} \frac{d \lambda(u)}{R_{u}}$.

Again, one may wonder whether the identity (2.4) holds at the level of increasing processes. However, the results in [3] rule out this possibility. It is then natural to ask for which functionals $\Phi: \mathcal{C}^{\uparrow} \rightarrow \mathbb{R}$ the identity

$$
\mathbb{E}\left(\Phi\left(H_{\sigma .}\right)\right)=\mathbb{E}\left(\Phi\left(\sigma_{a(\cdot)}\right)\right)
$$

may hold, where $\mathcal{C}^{\uparrow}$ stands for the space of càdlàg increasing paths $\omega: \mathbb{R}_{+} \rightarrow \mathbb{R}_{+}$. A partially positive response is provided by the following result.

Theorem 2.3. Consider a measurable function $\Gamma: \mathbb{R}_{+}^{3} \rightarrow \mathbb{R}_{+}$with $\Gamma(\cdot, 0, \cdot)=0$ and define

$$
\Phi(\omega)=\sum_{s \geq 0} \Gamma\left(\omega_{s-}, \Delta \omega_{s}, s\right), \quad \omega \in \mathcal{C}^{\uparrow}
$$

where $\Delta \omega_{s}=\omega_{s}-\omega_{s-}$. Then (2.5) holds.

More precisely, if $\Gamma(x, y, s)=f(x, s) g(y)$ for some measurable nonnegative functions $f$ and $g$ with $g(0)=0$, then

$$
\mathbb{E}\left(\Phi\left(H_{\sigma .}\right)\right)=\mathbb{E}\left(\Phi\left(\sigma_{a(\cdot)}\right)\right)=C(f) D(g),
$$


with

$$
C(f)=\int_{0}^{\infty} \frac{d \lambda}{\sqrt{1+\lambda^{2}}} \mathbb{E}\left[f\left(H_{\sigma_{\lambda}}, \lambda\right)\right]=\int_{0}^{\infty} \frac{d \lambda}{\sqrt{1+\lambda^{2}}} \mathbb{E}\left[f\left(\sigma_{a(\lambda)}, \lambda\right)\right]
$$

and

$$
D(g)=\int_{0}^{\infty} \frac{d t}{\sqrt{2 \pi t^{3}}} g(t) .
$$

We observe that Corollary 2.2 implies that the range of the subordinated clock $H_{\sigma}$ has the same distribution as the range of subordinator $\sigma$ (and hence is a regenerative set). In particular we see that (2.5) holds whenever for a generic increasing path $\omega, \Phi(\omega)$ depends only on the range of $\omega$. This provides a quick check of the identity $\mathbb{E}\left(\Phi\left(H_{\sigma .}\right)\right)=\mathbb{E}\left(\Phi\left(\sigma_{a(\cdot)}\right)\right)$ in the special case when the function $\Gamma$ does not depend on the time parameter, i.e., $\Gamma(x, y, s)=\Gamma(x, y)$.

2.5. An amplification and a variant of Theorem 2.3.

Theorem 2.4. Let $a, b \geq 0$ and let $\Gamma: \mathbb{R}_{+}^{2} \rightarrow \mathbb{R}_{+}$be a measurable function with $\Gamma(\cdot, 0)=0$. Introduce

$$
\mathcal{H}^{a, b}(\Gamma)(\ell)=\mathbb{E}\left[\sum_{\lambda \leq \ell}\left(R_{\sigma_{\lambda-}}\right)^{a} \frac{\Gamma\left(H_{\sigma_{\lambda-}}, H_{\sigma_{\lambda}}-H_{\sigma_{\lambda-}}\right)}{R_{\sigma_{\lambda}}^{b}}\right] .
$$

For $\Gamma=f \otimes g$ there holds

$$
\mathcal{H}^{a, b}(f \otimes g)(\ell)=h_{a-b}^{-}(f, \ell) h_{b}^{+}(g),
$$

where

$$
\begin{aligned}
h_{c}^{-}(f, \ell) & =\int_{0}^{\infty} d t f(t) \mathbb{E}\left[\frac{e^{(c+1) B_{t}}}{\sqrt{2 \pi A_{t}}}\left(1-e^{-\ell^{2} / 2 A_{t}}\right)\right] \\
h_{b}^{+}(g) & =\int_{0}^{\infty} \frac{d t g(t)}{\sqrt{2 \pi}} \mathbb{E}\left[\frac{e^{(2-b) B_{t}}}{A_{t}^{3 / 2}}\right] .
\end{aligned}
$$

It may be interesting to point out that these formulas simplify in the special case where $a=0$ and $b=1$. Indeed, using (1.4), one gets that

$$
h_{1}^{+}(g)=\int_{0}^{\infty} \frac{d t}{\sqrt{2 \pi t^{3}}} g(t)
$$

and then, using (1.3) and (1.2), that

$$
h_{-1}^{-}(f, \ell)=\int_{0}^{\infty} \frac{d t f(t)}{\sqrt{2 \pi t}}\left(1-a^{\prime}(\ell) \exp \left(-\frac{a^{2}(\ell)}{2 t}\right)\right)
$$

where $a$ and $a^{\prime}$ are defined below (1.2).

We also stress that the quantities

$$
m_{p, q}(t)=\mathbb{E}\left[\frac{\exp \left(p B_{t}\right)}{A_{t}^{q}}\right]
$$

arising in Theorem 2.4, have been studied in [6] and [7], [8]. 
2.6. A variant of Theorem 2.3 which involves the windings of planar Brownian motion. The following variant of Theorem 2.3 bears upon a relationship between the continuous winding process of planar Brownian motion, subordinated with $\left(\sigma_{\lambda}, \lambda \geq 0\right)$, and the standard Cauchy process.

Theorem 2.5. Let $Z_{u}=\left|Z_{u}\right| \exp \left(i \theta_{u}\right), u \geq 0$, denote a complex valued Brownian motion, starting from $1+i 0$, with $\left(\theta_{u}, u \geq 0\right)$ its continuous winding process. Let $\left(\sigma_{\lambda}, \lambda \geq 0\right)$ denote the inverse local time process of a linear Brownian motion, so $\sigma$ is a stable $(1 / 2)$ subordinator, which is assumed to be independent of $\left(Z_{u}, u \geq 0\right)$. Finally, let $\left(C_{\alpha}, \alpha \geq 0\right)$ be a standard Cauchy process.

For any measurable $\Gamma: \mathbb{R} \times \mathbb{R} \rightarrow \mathbb{R}_{+}$with $\Gamma(x, 0)=0$, we have

$$
\mathbb{E}\left[\sum_{\lambda \leq \ell} \Gamma\left(\theta_{\sigma_{\lambda-}}, \theta_{\sigma_{\lambda}}-\theta_{\sigma_{\lambda}-}\right)\right]=\mathbb{E}\left[\sum_{\lambda \leq \ell} \Gamma\left(C_{a(\lambda)-}, C_{a(\lambda)}-C_{a(\lambda)-}\right)\right]
$$

for all $\ell$ 's. In particular, for fixed $\ell \geq 0$, there is the equality in law:

$$
\theta_{\sigma_{\ell}} \stackrel{\text { (law) }}{=} C_{a(\ell)} \text {. }
$$

The reader interested in some applications of these identities in law to functionals of the winding process $\left(\theta_{u}, u \geq 0\right)$ may refer to Vakeroudis [16]. In particular, the identity (2.7) allows applying D. Williams' pinching method to yield yet another proof of Spitzer's celebrated theorem:

$$
\frac{2}{\log t} \theta_{t} \underset{t \rightarrow \infty}{\stackrel{\text { (law) }}{\longrightarrow}} C_{1} \text {. }
$$

2.7. The joint Laplace-Mellin transform of $\left(H_{\sigma_{\lambda}}, R_{\sigma_{\lambda}}\right)$. We now return to Theorem 2.3, or rather we discuss part of its proof, as given in Paragraph 3.2 below. A by-product of Lemma 4.1 is

$$
\mathbb{E}\left[\frac{1}{R_{\sigma_{\lambda}}} \mid H_{\sigma_{\lambda}}=h\right]=\frac{1}{\sqrt{1+\lambda^{2}}},
$$

an intriguing identity, which made us suspect that $R_{\sigma_{\lambda}}$ and $H_{\sigma_{\lambda}}$ might be independent. This is not the case, as we discovered by computing the joint Laplace-Mellin transform of $\left(H_{\sigma_{\lambda}}, R_{\sigma_{\lambda}}\right)$ :

Theorem 2.6. The following formulae hold:

$$
\begin{aligned}
& \mathbb{E}\left[\frac{1}{\left(R_{\sigma_{\lambda}}\right)^{2 b}}\right.\left.\exp \left(-\frac{\mu^{2}}{2} H_{\sigma_{\lambda}}\right)\right]=\mathbb{E}^{(\mu)}\left[\frac{1}{\left(R_{\sigma_{\lambda}}\right)^{2 b+\mu}}\right] \\
&=C_{b, \mu} \times \frac{F\left(\frac{\mu+1}{2}-b, \frac{\mu}{2}+1-b, \mu+1 ;-1 / \lambda^{2}\right)}{\left(1+\lambda^{2}\right)^{2 b-1 / 2}\left(\lambda^{2}\right)^{(\mu+1) / 2-b}},
\end{aligned}
$$

where $F \equiv{ }_{2} F_{1}$ denotes the classical three parameter family of hypergeometric functions, and $\mathbb{E}^{(\mu)}$ refers to the expectation with respect to the probability measure $\mathbb{P}^{(\mu)}$ under which $\left(R_{t}, t \geq 0\right)$ is a Bessel process with index $\mu$ (i.e., of dimension $\left.2+2 \mu\right)$ and $R_{0}=1$, and

$$
C_{b, \mu}=\frac{\Gamma\left(b+\frac{\mu}{2}+\frac{1}{2}\right) \Gamma\left(1+\frac{\mu}{2}-b\right)}{\Gamma\left(\frac{1}{2}\right) \Gamma(1+\mu)} .
$$


As a partial check of the formula (2.10), we have made explicit computations when $b=1 / 2, b=-\mu / 2$ (the result should be 1), and $b=0$ (the result is $\left.\exp (-\mu a(\lambda))=\left(\lambda+\sqrt{1+\lambda^{2}}\right)^{-\mu}\right)$. Let us give some details for $b=1 / 2$. We note that, for $b=1 / 2,(2.10)$ simplifies, as in the numerator

$$
F\left(\frac{\mu}{2}, \frac{\mu}{2}+\frac{1}{2}, \mu+1 ;-y\right)=\left(\frac{2}{1+\sqrt{1+y}}\right)^{\mu},
$$

and in the denominator

$$
\left(1+\lambda^{2}\right)^{1 / 2}\left(\lambda^{2}\right)^{\mu / 2} \equiv \lambda^{\mu}\left(1+\lambda^{2}\right)^{1 / 2} .
$$

Hence, using the fact that $C_{1 / 2, \mu}=2^{-\mu}$, formula (2.10) simplifies to

$$
2^{-\mu} \cdot \frac{2^{\mu}}{\left(1+\sqrt{1+1 / \lambda^{2}}\right)^{\mu}} \cdot \frac{1}{\lambda^{\mu} \sqrt{1+\lambda^{2}}} \equiv \frac{1}{\left(\sqrt{1+\lambda^{2}}+\lambda\right)^{\mu} \sqrt{1+\lambda^{2}}},
$$

which confirms the identity (2.9), since the right-hand side of the preceeding expression equals

$$
\frac{1}{\sqrt{1+\lambda^{2}}} \mathbb{E}\left(\exp \left(-\frac{\mu^{2}}{2} H_{\sigma_{\lambda}}\right)\right) \text {. }
$$

\section{Proof of Theorem 1.1}

3.1. We start by recalling some well-known facts about Brownian motion running up to an independent exponential time, which will be useful for the proof. In this subsection, $S_{p}$ denotes an exponential random variable with parameter $p>0$, independent of the Brownian motion $B$. For $t \geq 0$, let $g_{t}=\sup \left\{u<t: B_{u}=0\right\}$ be the last zero of $B$ before $t$. It is known that the processes $\left(B_{u}, u \leq g_{S_{p}}\right)$ and $\left(B_{g_{S_{p}}+u}, u \leq S_{p}-g_{S_{p}}\right)$ are independent. As a consequence, the variables $L_{S_{p}}\left(\equiv L_{g_{S_{p}}}\right)$ and $B_{S_{p}}$ are independent. Moreover, since $L_{t}$ and $\left|B_{t}\right|$ have the same law (see (2.2)), the same applies to $L_{S_{p}}$ and $\left|B_{S_{p}}\right|$. Their common density is

$$
\sqrt{2 p} \exp (-\sqrt{2 p} u), \quad u \geq 0
$$

(this is because $\mathbb{P}\left(L_{S_{p}} \geq \ell\right)=\mathbb{P}\left(S_{p} \geq \tau_{\ell}\right)=\mathbb{E}\left[\exp \left(-p \tau_{\ell}\right)\right]=\exp (-\ell \sqrt{2 p})$, if $\tau_{\ell}$ is the time $L$. reaches $\ell$ ). An equivalent way to express this property is

$$
\sqrt{2 \mathbf{e}}(|\beta(1)|, \lambda(1)) \stackrel{(\text { law })}{=}\left(\mathbf{e}, \mathbf{e}^{\prime}\right),
$$

where on the left $\mathbf{e} \stackrel{\text { (law) }}{=} S_{1}$ is independent of $\beta$, and on the right the two variables are independent copies of $S_{1}$.

3.2. Recall the discussion in Paragraph 2.1. Our main goal is to show that

$$
\left(\sinh \left(\left|B_{t}\right|\right), \sinh \left(L_{t}\right)\right) \stackrel{(\text { law })}{=}\left(\exp \left(-B_{t}\right) \sqrt{A_{t}}|\beta(1)|, \sqrt{A_{t}} \lambda(1)\right)
$$

where $B$ and $\beta$ are independent Brownian motions. 
Note from the scaling property that (3.1) is equivalent to the identity in distribution

$$
\left(\sinh \left(\left|B_{t}\right|\right), \sinh \left(L_{t}\right)\right) \stackrel{(\text { law })}{=}\left(\exp \left(-B_{t}\right)\left|\beta\left(A_{t}\right)\right|, \lambda\left(A_{t}\right)\right) .
$$

We will establish (3.1) by computing the joint Mellin transforms of either side, but before doing so we replace $t$ with an exponential time $S_{p}$ and multiply both sides by $\sqrt{2 \mathbf{e}}$, assuming implicitly that $S_{p}, \mathbf{e}, B$ and $\beta$ are independent. What will be proved is:

$$
\sqrt{2 \mathbf{e}}\left(\sinh \left(\left|B_{S_{p}}\right|\right), \sinh \left(L_{S_{p}}\right)\right) \stackrel{(\text { law })}{=} \sqrt{2 \mathbf{e}}\left(\exp \left(-B_{S_{p}}\right) \sqrt{A_{S_{p}}}|\beta(1)|, \sqrt{A_{S_{p}}} \lambda(1)\right) .
$$

From the one-dimensional Bougerol identity (1.1), the left-hand side of (3.2) has the same distribution as

$$
\sqrt{2 \mathbf{e}}\left(|N| \sqrt{A_{S_{p}}},\left|N^{\prime}\right| \sqrt{A_{S_{p}^{\prime}}^{\prime}}\right),
$$

where $N$ and $N^{\prime}$ are independent standard normals and $A^{\prime}$ is a copy of $A$ which is also independent of the other quantities. On the right-hand side of (3.2), use the facts in Paragraph 3.1 and in particular the elementary identity

$$
\mathbf{e} \stackrel{(\text { law })}{=}|N| \sqrt{2 \mathbf{e}}
$$

(see also formula (4.8.1) in [5]) to obtain

$$
\begin{gathered}
\sqrt{2 \mathbf{e}}\left(\exp \left(-B_{S_{p}}\right) \sqrt{A_{S_{p}}}|\beta(1)|, \sqrt{A_{S_{p}}} \lambda(1)\right) \stackrel{(\text { law })}{=}\left(\exp \left(-B_{S_{p}}\right) \sqrt{A_{S_{p}}} \mathbf{e}, \sqrt{A_{S_{p}}} \mathbf{e}^{\prime}\right) \\
\stackrel{(\text { law })}{=}\left(\exp \left(-B_{S_{p}}\right) \sqrt{A_{S_{p}}}|N| \sqrt{2 \mathbf{e}}, \sqrt{A_{S_{p}}}\left|N^{\prime}\right| \sqrt{2 \mathbf{e}^{\prime}}\right) .
\end{gathered}
$$

Squaring, we are left with calculating the joint Mellin transforms of

$$
\mathbf{e}\left(A_{S_{p}}, A_{S_{p}^{\prime}}^{\prime}\right) \quad \text { and } \quad\left(\exp \left(-2 B_{S_{p}}\right) A_{S_{p}} \mathbf{e}, A_{S_{p}} \mathbf{e}^{\prime}\right)
$$

and verifying that they are equal (the $N$ and $N^{\prime}$ on both sides of (3.3) and (3.4) can be cancelled).

The essential ingredient for computing these Mellin transforms is

$$
A_{S_{p}}^{(\nu)} \stackrel{(\text { law })}{=} \frac{\beta_{1, a}}{2 \gamma_{b}}
$$

(see [18], paper no. 6, p.94), where $A^{(\nu)}$ denotes the exponential functional

$$
A_{t}^{(\nu)}=\int_{0}^{t} d s \exp \left(2 B_{s}^{(\nu)}\right), \quad t \geq 0,
$$

of a Brownian motion with drift $\nu, B_{s}^{(\nu)}=B_{s}+\nu s, \beta_{u, v}$ is a beta variable with parameters $(u, v), \gamma_{b}$ is a gamma variable with parameter $b$, and

$$
a=a(\nu, p)=\frac{1}{2}\left(\nu+\sqrt{2 p+\nu^{2}}\right), \quad b=b(\nu, p)=\frac{1}{2}\left(-\nu+\sqrt{2 p+\nu^{2}}\right) .
$$


The Mellin transform of $A_{S_{p}}^{(\nu)}$ is then

$$
\mathbb{E}\left(A_{S_{p}}^{(\nu)}\right)^{r}=2^{-r} \frac{\Gamma(1+a) \Gamma(1+r) \Gamma(b-r)}{\Gamma(1+a+r) \Gamma(b)} .
$$

On the one hand, since $a(0, p)=b(0, p)=\sqrt{p / 2}$,

$$
\begin{aligned}
& \mathbb{E}\left[\left(\mathbf{e} A_{S_{p}}\right)^{c}\left(\mathbf{e} A_{S_{p}^{\prime}}^{\prime}\right)^{d}\right] \\
& \quad=2^{-c-d} \frac{\Gamma(1+c+d) \Gamma(1+a) \Gamma(1+c) \Gamma(b-c) \Gamma(1+a) \Gamma(1+d) \Gamma(b-d)}{\Gamma(1+a+c) \Gamma(1+a+d) \Gamma(b)^{2}} \\
& \quad=2^{-c-d} \frac{p}{2} \frac{\Gamma(1+c+d) \Gamma(1+c) \Gamma\left(\sqrt{\frac{p}{2}}-c\right) \Gamma(1+d) \Gamma\left(\sqrt{\frac{p}{2}}-d\right)}{\Gamma\left(1+c+\sqrt{\frac{p}{2}}\right) \Gamma\left(1+d+\sqrt{\frac{p}{2}}\right)} .
\end{aligned}
$$

On the other hand, recalling the definition of $A_{t}^{(\nu)}$ and using the Girsanov-Cameron-Martin theorem, we obtain

$$
\begin{aligned}
\mathbb{E}\left[\left(\exp \left(-2 B_{S_{p}}\right) A_{S_{p}} \mathbf{e}\right)^{c}\left(A_{S_{p}} \mathbf{e}^{\prime}\right)^{d}\right] & =\Gamma(1+c) \Gamma(1+d) \mathbb{E}\left[\exp \left(-2 c B_{S_{p}}\right)\left(A_{S_{p}}\right)^{c+d}\right] \\
& =\Gamma(1+c) \Gamma(1+d) \mathbb{E}\left[\exp \left(2 c^{2} S_{p}\right)\left(A_{S_{p}}^{(-2 c)}\right)^{c+d}\right] .
\end{aligned}
$$

From the elementary identity

$$
\mathbb{P}\left(S_{p} \in d s ; \exp \left(\eta S_{p}\right)\right)=\frac{p}{p-\eta} \mathbb{P}\left(S_{p-\eta} \in d s\right), \quad \eta<p
$$

we find, letting $q=p-2 c^{2}$,

$$
\begin{aligned}
\mathbb{E} & {\left[\left(\exp \left(-2 B_{S_{p}}\right) A_{S_{p}} \mathbf{e}\right)^{c}\left(A_{S_{p}} \mathbf{e}^{\prime}\right)^{d}\right] } \\
& =\frac{p}{q} \Gamma(1+c) \Gamma(1+d) \mathbb{E}\left[\left(A_{S_{q}}^{(-2 c)}\right)^{c+d}\right] \\
& =2^{-c-d} \frac{p}{q} \Gamma(1+c) \Gamma(1+d) \frac{\Gamma(1+a(-2 c, q)) \Gamma(1+c+d) \Gamma(b(-2 c, q)-c-d)}{\Gamma(1+a(-2 c, q)+c+d) \Gamma(b(-2 c, q))} .
\end{aligned}
$$

Now, $a(-2 c, q)=-c+\sqrt{p / 2}, b(-2 c, q)=c+\sqrt{p / 2}$, and thus, comparing $(3.7)$ and the preceding,

$$
\begin{gathered}
\frac{p}{q} \frac{\Gamma(1+a(-2 c, q)) \Gamma(b(-2 c, q)-c-d)}{\Gamma(1+a(-2 c, q)+c+d) \Gamma(b(-2 c, q))}=\frac{p}{p-2 c^{2}} \frac{\Gamma\left(1-c+\sqrt{\frac{p}{2}}\right) \Gamma\left(-d+\sqrt{\frac{p}{2}}\right)}{\Gamma\left(1+d+\sqrt{\frac{p}{2}}\right) \Gamma\left(c+\sqrt{\frac{p}{2}}\right)} \\
=\frac{p}{p-2 c^{2}}\left(\sqrt{\frac{p}{2}}-c\right)\left(\sqrt{\frac{p}{2}}+c\right) \frac{\Gamma\left(\sqrt{\frac{p}{2}}-c\right) \Gamma\left(\sqrt{\frac{p}{2}}-d\right)}{\Gamma\left(1+c+\sqrt{\frac{p}{2}}\right) \Gamma\left(1+d+\sqrt{\frac{p}{2}}\right)} \\
=\frac{p}{2} \frac{\Gamma\left(\sqrt{\frac{p}{2}}-c\right) \Gamma\left(\sqrt{\frac{p}{2}}-d\right)}{\Gamma\left(1+c+\sqrt{\frac{p}{2}}\right) \Gamma\left(1+d+\sqrt{\frac{p}{2}}\right)} .
\end{gathered}
$$

Note that, along the way, it was necessary to assume $q=p-2 c^{2}>0$, so that $c$ needed to be taken small enough, and likewise for $d$; precisely, $c, d<\sqrt{p / 2}$. However, even with these restrictions, we can conclude the proof of the identity in law of the two vectors in (3.5), thus ending the proof of (3.1). 
3.3. The second identity in Theorem 1.1 may be proved rather simply, by first noting that (from the scaling property and the independence of $B$ and $\beta$ )

$$
\left(\beta\left(A_{t}\right), \exp \left(-B_{t}\right) \lambda\left(A_{t}\right)\right) \stackrel{\text { law })}{=}\left(\sqrt{A_{t}} \beta(1), \exp \left(-B_{t}\right) \sqrt{A_{t}} \lambda(1)\right)
$$

and then recalling (from the proof of Bougerol's identity in [1]) that time reversal

$$
\left(B_{t}-B_{(t-u)}, 0 \leq u \leq t\right) \stackrel{(\text { law })}{=}\left(B_{u}, 0 \leq u \leq t\right)
$$

implies

$$
\left(A_{t}, \exp \left(-2 B_{t}\right) A_{t}\right) \stackrel{(\text { law })}{=}\left(\exp \left(-2 B_{t}\right) A_{t}, A_{t}\right) .
$$

This completes the proof of Theorem 1.1.

\section{Proofs of Theorems 2.3 to 2.6}

4.1. Proof of Theorem 2.3. (a) A key for the proof of Theorem 2.3 is the following interesting, and puzzling, identity, as discussed in Subsection 2.7.

Lemma 4.1. For any measurable function $f: \mathbb{R}_{+} \mapsto \mathbb{R}_{+}$, and any $s \geq 0$, we have:

$$
\mathbb{E}\left[\frac{1}{R_{\sigma_{s}}} f\left(H_{\sigma_{s}}\right)\right]=\frac{1}{\sqrt{1+s^{2}}} \mathbb{E}\left[f\left(\sigma_{a(s)}\right)\right] .
$$

Proof. For all $q, t, \varepsilon>0$, we have from (2.4) that

$$
\mathbb{E}\left(\exp \left(-q \sigma_{a(t)}\right)-\exp \left(-q \sigma_{a(t+\varepsilon)}\right)\right)=\mathbb{E}\left(\exp \left(-q H_{\sigma_{t}}\right)-\exp \left(-q H_{\sigma_{t+\varepsilon}}\right)\right) .
$$

The left-hand side can be computed explicitly and we obtain

$\exp (-a(t) \sqrt{2 q})-\exp (-a(t+\varepsilon) \sqrt{2 q}) \sim \frac{\varepsilon}{\sqrt{1+t^{2}}} \sqrt{2 q} \exp (-a(t) \sqrt{2 q}), \quad \varepsilon \rightarrow 0$.

We next turn our attention to the right-hand side and apply the Markov property. In this direction, it is convenient to introduce a two-dimensional Bessel process $R^{\prime}$ which is independent of $R$ and write $H^{\prime}$ for its clock. Likewise, $\sigma^{\prime}$ refers to an independent subordinator which has the same distribution as $\sigma$. For every $r>0$, the notation $\mathbb{P}_{r}^{\prime}$ refers to the law under which $R_{0}^{\prime}=r$ and $\mathbb{E}_{r}^{\prime}$ to the mathematical expectation under $\mathbb{P}_{r}^{\prime}$. We point out that the scaling property implies the equalities

$$
\mathbb{E}_{r}^{\prime}\left(1-\exp \left(-q H_{\sigma_{\varepsilon}^{\prime}}^{\prime}\right)\right)=\mathbb{E}_{1}^{\prime}\left(1-\exp \left(-q H_{r^{-2} \sigma_{\varepsilon}^{\prime}}^{\prime}\right)\right)=\mathbb{E}_{1}^{\prime}\left(1-\exp \left(-q H_{\sigma_{\varepsilon / r}^{\prime}}^{\prime}\right)\right) .
$$

Of course we can also express the right-hand side as $\mathbb{E}\left(1-\exp \left(-q H_{\sigma_{\varepsilon / r}}\right)\right)$. Writing $\sigma_{t+\varepsilon}=\sigma_{t}+\sigma_{\varepsilon}^{\prime}$, with $\sigma_{\varepsilon}^{\prime}$ independent of $\sigma_{t}$ and $R$, we get from an application of the Markov property,

$$
\begin{aligned}
\mathbb{E}\left(\exp \left(-q H_{\sigma_{t}}\right)-\exp \left(-q H_{\sigma_{t+\varepsilon}}\right)\right) & =\mathbb{E}\left(\exp \left(-q H_{\sigma_{t}}\right) \mathbb{E}_{R_{\sigma_{t}}}^{\prime}\left(1-\exp \left(-q H_{\sigma_{\varepsilon}^{\prime}}^{\prime}\right)\right)\right) \\
& =\mathbb{E}\left(\exp \left(-q H_{\sigma_{t}}\right) \mathbb{E}_{1}^{\prime}\left(1-\exp \left(-q H_{\sigma_{\varepsilon / R_{\sigma_{t}}}^{\prime}}^{\prime}\right)\right)\right) \\
& =\mathbb{E}\left(\exp \left(-q H_{\sigma_{t}}\right)\left(1-\exp \left\{-a\left(\varepsilon / R_{\sigma_{t}}\right) \sqrt{2 q}\right\}\right)\right),
\end{aligned}
$$


where the third equality follows from (2.4). Note that when $\varepsilon \rightarrow 0+$, the preceding quantity is equivalent to

$$
\varepsilon \sqrt{2 q} \mathbb{E}\left(\exp \left(-q H_{\sigma_{t}}\right) \frac{1}{R_{\sigma_{t}}}\right)
$$

Putting the pieces together, we arrive at

$$
\mathbb{E}\left(\exp \left(-q H_{\sigma_{t}}\right) \frac{1}{R_{\sigma_{t}}}\right)=\frac{1}{\sqrt{1+t^{2}}} \exp (-a(t) \sqrt{2 q})=\frac{1}{\sqrt{1+t^{2}}} \mathbb{E}\left(\exp \left(-q \sigma_{a(t)}\right)\right)
$$

for all $t, q>0$, which establishes Lemma 4.1.

(b) To conclude the proof of Theorem 2.3, define, for jump intensity measures, the following notation:

$$
\begin{aligned}
\mathcal{H}(\Gamma)(s) & =\mathbb{E}\left[\sum_{\lambda \leq s} \Gamma\left(H_{\sigma_{\lambda^{-}}}, H_{\sigma_{\lambda}}-H_{\sigma_{\lambda^{-}}}\right)\right] \\
\mathcal{K}(\Gamma)(s) & =\mathbb{E}\left[\sum_{\alpha \leq a(s)} \Gamma\left(\sigma_{\alpha^{-}}, \sigma_{\alpha}-\sigma_{\alpha^{-}}\right)\right]
\end{aligned}
$$

for given $s$, and Borel $\Gamma: \mathbb{R}_{+} \times \mathbb{R}_{+} \rightarrow \mathbb{R}_{+}$such that $\Gamma(x, 0)=0$. Then, in order to finish the proof of Theorem 2.3, it suffices to take $\Gamma=f \otimes g$ and to show

$$
\begin{aligned}
& \mathcal{H}(f \otimes g)(s)=h(f)(s) \int_{0}^{\infty} \frac{d t}{\sqrt{2 \pi t^{3}}} g(t), \\
& \mathcal{K}(f \otimes g)(s)=k(f)(s) \int_{0}^{\infty} \frac{d t}{\sqrt{2 \pi t^{3}}} g(t),
\end{aligned}
$$

with, furthermore, the quantities $h(f)(s)$ and $k(f)(s)$ being equal, and equal to:

$$
\begin{aligned}
h(f)(s) & =\int_{0}^{s} d \lambda \mathbb{E}\left[\frac{1}{R_{\sigma_{\lambda}}} f\left(H_{\sigma_{\lambda}}\right)\right]=\int_{0}^{s} \frac{d \lambda}{\sqrt{1+\lambda^{2}}} \mathbb{E}\left[f\left(H_{\sigma_{\lambda}}\right)\right] \\
\| & \\
k(f)(s) & =\int_{0}^{a(s)} d \alpha \mathbb{E}\left[f\left(\sigma_{\alpha}\right)\right]=\int_{0}^{s} \frac{d \lambda}{\sqrt{1+\lambda^{2}}} \mathbb{E}\left[f\left(\sigma_{a(\lambda)}\right)\right] .
\end{aligned}
$$

Now, concerning $\mathcal{K}(\Gamma)(s)$, since the Lévy measure of the subordinator $\left(\sigma_{\alpha}, \alpha \geq 0\right)$ is $d t / \sqrt{2 \pi t^{3}}$, we have

$$
\begin{aligned}
\mathcal{K}(\Gamma)(s) & =\mathbb{E}\left[\int_{0}^{a(s)} d \alpha f\left(\sigma_{\alpha-}\right)\right] \int_{0}^{\infty} \frac{d t}{\sqrt{2 \pi t^{3}}} g(t) \\
& =\int_{0}^{s} \frac{d u}{\sqrt{1+u^{2}}} \mathbb{E}\left[f\left(\sigma_{a(u)}\right)\right] \int_{0}^{\infty} \frac{d t}{\sqrt{2 \pi t^{3}}} g(t) .
\end{aligned}
$$


Concerning $\mathcal{H}(\Gamma)(s)$, starting again with the same argument (i.e., the knowledge of the Lévy measure of $\left.\left(\sigma_{u}, u \geq 0\right)\right)$, we obtain

$$
\begin{aligned}
\mathcal{H}(\Gamma)(s) & =\mathbb{E}\left[\int_{0}^{s} d \lambda \int_{0}^{\infty} \frac{d t}{\sqrt{2 \pi t^{3}}} f\left(H_{\sigma_{\lambda}}\right) g\left(H_{\sigma_{\lambda}+t}-H_{\sigma_{\lambda}}\right)\right] \\
& =\mathbb{E}\left[\int_{0}^{s} d \lambda \int_{0}^{\infty} \frac{d t}{\sqrt{2 \pi t^{3}}} f\left(H_{\sigma_{\lambda}}\right) \mathbb{E}_{R_{\sigma_{\lambda}}^{\prime}}\left(g\left(H_{t}^{\prime}\right)\right)\right]
\end{aligned}
$$

(from the Markov property for $R$ ). However, by scaling, we have

$$
\mathbb{E}_{\rho}^{\prime}\left[g\left(H_{t}^{\prime}\right)\right]=\mathbb{E}\left[g\left(H_{t / \rho^{2}}\right)\right]
$$

so that, substituting (4.3) into (4.2), we obtain

$$
\int_{0}^{\infty} \frac{d t}{\sqrt{2 \pi t^{3}}} \mathbb{E}_{\rho}^{\prime}\left[g\left(H_{t}^{\prime}\right)\right]=\int_{0}^{\infty} \frac{d t}{\sqrt{2 \pi t^{3}}} \mathbb{E}\left[g\left(H_{t / \rho^{2}}\right)\right]=\frac{1}{\rho} \int_{0}^{\infty} \frac{d u}{\sqrt{2 \pi u^{3}}} \mathbb{E}\left[g\left(H_{u}\right)\right]
$$

Note that, since the inverse of $\left\{u \rightarrow H_{u}\right\}$ is: $t \rightarrow A_{t}=\int_{0}^{t} d v e^{2 B_{v}}$, we have

$$
\int_{0}^{\infty} \frac{d u}{\sqrt{2 \pi u^{3}}} \mathbb{E}\left[g\left(H_{u}\right)\right]=\frac{1}{\sqrt{2 \pi}} \mathbb{E}\left[\int_{0}^{\infty} d t g(t) \frac{e^{2 B_{t}}}{\sqrt{A_{t}^{3}}}\right]=\int_{0}^{\infty} d t g(t) \frac{1}{\sqrt{2 \pi t^{3}}}
$$

by (1.4).

Returning to (4.2), we have obtained

$$
\mathcal{H}(\Gamma)(s)=\mathbb{E}\left[\int_{0}^{s} d \lambda \frac{1}{R_{\sigma_{\lambda}}} f\left(H_{\sigma_{\lambda}}\right)\right] \int_{0}^{\infty} \frac{d t}{\sqrt{2 \pi t^{3}}} g(t) .
$$

c) Finally, to obtain the equality between $\mathcal{H}(\Gamma)(s)$ and $\mathcal{K}(\Gamma)(s)$, there remains to show, with the notation as in the statement of Theorem 2.4, that

$$
h(f)(s)=k(f)(s) \quad \text { for every Borel } f \geq 0 .
$$

Again, it suffices to prove this for $f_{\lambda}(a)=e^{-\lambda a}$, for any $\lambda \geq 0$. Now we have

$$
\begin{aligned}
\mathbb{E}\left[\exp \left(-\nu H_{\sigma_{s}}\right)\right] & =1+\mathbb{E}\left[\sum_{\theta \leq s}\left(e^{-\nu H_{\sigma_{\lambda}}}-e^{-\nu H_{\sigma_{\lambda}-}}\right)\right] \\
& =1+\mathbb{E}\left[\sum_{\theta \leq s} e^{-\nu H_{\sigma_{\lambda}-}}\left(e^{-\nu\left(H_{\sigma_{\lambda}}-H_{\sigma_{\lambda^{-}}}\right)}-1\right)\right] \\
& =1+\mathcal{H}\left(f_{\nu} \otimes g_{\nu}\right)(s) \\
(\text { where } & \left.: f_{\nu}(a)=\exp (-\nu a) ; g_{\nu}(b)=\left(e^{-\nu b}-1\right)\right) \\
& =1+h\left(f_{\nu}\right)(s)\left(\int_{0}^{\infty} \frac{d t}{\sqrt{2 \pi t^{3}}} g_{\nu}(t)\right) .
\end{aligned}
$$

On the other hand,

$$
\mathbb{E}\left[\exp \left(-\nu \sigma_{a(s)}\right)\right]=1+\int_{0}^{a(s)} d \alpha f_{\nu}(\alpha) \int_{0}^{\infty} \frac{d t}{\sqrt{2 \pi t^{3}}} g_{\nu}(t) .
$$


Thus, explicitly,

$$
\mathbb{E}\left[\exp \left(-\nu H_{\sigma_{s}}\right)\right]=1-\left(h\left(f_{\nu}\right)(s)\right) \sqrt{2 \nu}
$$

whereas

$$
\mathbb{E}\left[\exp \left(-\nu \sigma_{a(s)}\right)\right]=1-k\left(f_{\nu}\right)(s) \sqrt{2 \nu} .
$$

Since the left-hand sides of (4.6) and (4.7) are equal, so are the right-hand sides. Therefore, for all Borel $f \geq 0$,

$$
h(f)(s)=k(f)(s) .
$$

Hence, in complete generality,

$$
\mathcal{H}(\Gamma)(s)=\mathcal{K}(\Gamma)(s),
$$

which finishes the proof of Theorem 2.3.

4.2. Proof of Theorem 2.4. Here are the main steps of this proof, which is quite similar to that of Theorem 2.3:

1) We first transform

$$
\begin{aligned}
\mathcal{H}^{a, b}(\Gamma)(\ell) & =\mathbb{E}\left[\sum_{\lambda \leq \ell}\left(R_{\sigma_{\lambda-}}\right)^{a} f\left(H_{\sigma_{\lambda-}}\right) \frac{g\left(H_{\sigma_{\lambda}}-H_{\sigma_{\lambda-}}\right) 1_{\left(\sigma_{\lambda}>\sigma_{\lambda}-\right)}}{\left(R_{\sigma_{\lambda}}\right)^{b}}\right] \\
& =\mathbb{E}\left[\int_{0}^{\ell} d \lambda\left(R_{\sigma_{\lambda}}\right)^{a} f\left(H_{\sigma_{\lambda}}\right) \int_{0}^{\infty} \frac{d t}{\sqrt{2 \pi t^{3}}} \frac{g\left(H_{\sigma_{\lambda}+t}-H_{\sigma_{\lambda}}\right)}{\left(R_{\sigma_{\lambda}+t}\right)^{b}}\right] \\
& =\mathbb{E}\left[\int_{0}^{\ell} d \lambda\left(R_{\sigma_{\lambda}}\right)^{a} f\left(H_{\sigma_{\lambda}}\right) E_{R_{\sigma_{\lambda}}}\left[\int_{0}^{\infty} \frac{d t}{\sqrt{2 \pi t^{3}}} \frac{g\left(H_{t}\right)}{\left(R_{t}\right)^{b}}\right]\right]
\end{aligned}
$$

We begin by studying

$$
\begin{aligned}
h^{(+)}(r, g) & =\mathbb{E}_{r}\left[\int_{0}^{\infty} \frac{d t}{\sqrt{2 \pi t^{3}}} \frac{g\left(H_{t}\right)}{R_{t}^{b}}\right]=\mathbb{E}\left[\int_{0}^{\infty} \frac{d t}{\sqrt{2 \pi t^{3}}} \frac{g\left(H_{t / r^{2}}\right)}{\left(r R_{t / r^{2}}\right)^{b}}\right] \text { (by scaling) } \\
& =\frac{1}{r^{b}} \mathbb{E}\left[\int_{0}^{\infty} \frac{d t}{\sqrt{2 \pi t^{3}}} \frac{g\left(H_{t / r^{2}}\right)}{\left(R_{t / r^{2}}\right)^{b}}\right]=\frac{1}{r^{b}}\left(\frac{1}{r}\right) \mathbb{E}\left[\int_{0}^{\infty} \frac{d u}{\sqrt{2 \pi u^{3}}} \frac{g\left(H_{u}\right)}{\left(R_{u}\right)^{b}}\right] \\
& \equiv \frac{1}{r^{b+1}} h_{b}^{(+)}(g) .
\end{aligned}
$$

We then study:

$$
\begin{aligned}
h_{b}^{(+)}(g) & =\mathbb{E}\left[\int_{0}^{\infty} \frac{d u}{\sqrt{2 \pi u^{3}}} \frac{g\left(H_{u}\right)}{\left(R_{u}\right)^{b}}\right]=\mathbb{E}\left[\int_{0}^{\infty} \frac{d t e^{2 B_{t}}}{\sqrt{2 \pi A_{t}^{3}}} \frac{g(t)}{\left(\exp \left(b B_{t}\right)\right)}\right] \\
& =\mathbb{E}\left[\int_{0}^{\infty} \frac{d t e^{(2-b) B_{t}}}{\sqrt{2 \pi A_{t}^{3}}} g(t)\right]=\int_{0}^{\infty} \frac{d t g(t)}{\sqrt{2 \pi}} \mathbb{E}\left[\frac{e^{(2-b) B_{t}}}{\sqrt{A_{t}^{3}}}\right] \\
& =\int_{0}^{\infty} \frac{d t g(t)}{\sqrt{2 \pi}} m_{2-b, 3 / 2}(t)
\end{aligned}
$$

where the quantity $m_{p, q}(t)$ has been defined in (2.6). 
2) Let us return to

$$
\begin{aligned}
\mathcal{H}^{a, b}(\Gamma)(\ell) & =\mathbb{E}\left[\int_{0}^{\ell} d \lambda\left(R_{\sigma_{\lambda}}\right)^{a-b-1} f\left(H_{\sigma_{\lambda}}\right)\right] h_{b}^{(+)}(g) \\
& =h_{a-b}^{(-)}(f, \ell) h_{b}^{(+)}(g), \quad \text { for } F=f \otimes g .
\end{aligned}
$$

Thus, our next aim is to study

$$
h_{c}^{(-)}(f, \ell):=\mathbb{E}\left[\int_{0}^{\ell} d \lambda\left(R_{\sigma_{\lambda}}\right)^{c-1} f\left(H_{\sigma_{\lambda}}\right)\right] .
$$

We can re-express this quantity as

$$
\begin{aligned}
h_{c}^{(-)}(f, \ell) & =\mathbb{E}\left[\int_{0}^{\tau_{\ell}} d L_{u}\left(R_{u}\right)^{c-1} f\left(H_{u}\right)\right] \\
& =\int_{0}^{\infty} \frac{d u}{\sqrt{2 \pi u}} \mathbb{P}\left(L_{u}<\ell \mid B_{u}=0\right) \mathbb{E}\left[\left(R_{u}\right)^{c-1} f\left(H_{u}\right)\right] \\
& =\int_{0}^{\infty} \frac{d u}{\sqrt{2 \pi u}} \mathbb{P}(\sqrt{u} \sqrt{2 \mathbf{e}}<\ell) \mathbb{E}\left[\left(R_{u}\right)^{c-1} f\left(H_{u}\right)\right] \\
& =\int_{0}^{\infty} \frac{d u}{\sqrt{2 \pi u}} \mathbb{P}\left(\mathbf{e} \leq \ell^{2} / 2 u\right) \mathbb{E}\left[\left(R_{u}\right)^{c-1} f\left(H_{u}\right)\right] \\
& =\int_{0}^{\infty} \frac{d u}{\sqrt{2 \pi u}}\left(1-\exp \left(-\ell^{2} / 2 u\right)\right) \mathbb{E}\left[\left(R_{u}\right)^{c-1} f\left(H_{u}\right)\right] \\
& =\mathbb{E}\left[\int_{0}^{\infty} \frac{d A_{t}}{\sqrt{2 \pi A_{t}}}\left(1-e^{-\ell^{2} / 2 A_{t}}\right) \exp \left((c-1) B_{t}\right) f(t)\right] \\
& =\mathbb{E}\left[\int_{0}^{\infty} d t \frac{e^{(c+1) B_{t}}}{\sqrt{2 \pi A_{t}}}\left(1-e^{-\ell^{2} / 2 A_{t}}\right) f(t)\right] \\
& =\int_{0}^{\infty} d t f(t) \mathbb{E}\left[\frac{e^{(c+1) B_{t}}}{\sqrt{2 \pi A_{t}}}\left(1-e^{-\ell^{2} / 2 A_{t}}\right)\right] .
\end{aligned}
$$

4.3. Proof of Theorem 2.5. Theorem 2.5 is a simple consequence of Theorem 2.3, once one uses the well-known skew-product representation of $\theta_{t}=\gamma_{H_{t}}$, where $\left(\gamma_{u}, u \geq 0\right)$ is a real-valued Brownian motion independent of $\left(H_{t}, t \geq 0\right)$ (we already gave some references before Corollary 2.1). All one needs to do is to "freeze" $\gamma$, then apply Theorem 2.3, and finally use Spitzer's representation of the Cauchy process as

$$
\left(C_{\alpha}, \alpha \geq 0\right) \stackrel{(\text { law })}{=}\left(\gamma_{\sigma_{\alpha}}, \alpha \geq 0\right) .
$$

4.4. Proof of Theorem 2.6. The first equality follows from the (local) absolute continuity relationship between the laws of different Bessel processes, see, e.g., [18].

There remains to prove the second equality. For this purpose, we use the same arguments as in the proof of (16) in [9]; some details follow.

Let

$$
J \stackrel{\text { def }}{=} \mathbb{E}^{(\mu)}\left[\frac{1}{\left(R_{\sigma_{\lambda}}\right)^{2 b+\mu}}\right]=\frac{1}{\Gamma\left(b+\frac{\mu}{2}\right)} \int_{0}^{\infty} d u u^{b+\mu / 2-1} \mathbb{E}^{(\mu)}\left[\exp \left(-u R_{\sigma_{\lambda}}^{2}\right)\right] .
$$


There is a classical expression for $\mathbb{E}^{(\mu)}\left[\exp \left(-u R_{t}^{2}\right)\right]$ (see, e.g., page 441 of $[15]$ ), which yields

$$
\mathbb{E}^{(\mu)}\left[\exp \left(-u R_{\sigma_{\lambda}}^{2}\right)\right]=\mathbb{E}\left(\left(1+2 u \sigma_{\lambda}\right)^{-1-\mu} \exp \left(-\frac{u}{1+2 u \sigma_{\lambda}}\right)\right) .
$$

Using Fubini and making the change of variables $v=2 u \sigma_{\lambda} /\left(1+2 u \sigma_{\lambda}\right)$, we obtain the following expression for the integral

$$
\begin{aligned}
I & \stackrel{\text { def }}{=} \int_{0}^{\infty} d u u^{b+\mu / 2-1}\left(1+2 u \sigma_{\lambda}\right)^{-1-\mu} \exp \left(-\frac{u}{1+2 u \sigma_{\lambda}}\right) \\
& =\frac{1}{\left(2 \sigma_{\lambda}\right)^{b+\mu / 2}} \int_{0}^{1} d v v^{b+\mu / 2-1}(1-v)^{\mu / 2-b} \exp \left(-\frac{v}{2 \sigma_{\lambda}}\right) .
\end{aligned}
$$

Hence, using Fubini again, we obtain

$$
J=\frac{1}{\Gamma(b+\mu / 2)} \int_{0}^{1} d v v^{b+\mu / 2-1}(1-v)^{\mu / 2-b} \mathbb{E}\left[\frac{1}{\left(2 \sigma_{\lambda}\right)^{b+\mu / 2}} \exp \left(-\frac{v}{2 \sigma_{\lambda}}\right)\right]
$$

To compute this last expectation, which we denote by $K$, we use

$$
\frac{1}{2 \sigma_{\lambda}} \stackrel{\text { (law) }}{=} \frac{N^{2}}{2 \lambda^{2}} \stackrel{\text { (law) }}{=} \frac{\gamma_{1 / 2}}{\lambda^{2}}
$$

where $\gamma_{1 / 2}$ is a standard gamma(1/2)-variable. Then we obtain

$$
K=\frac{\Gamma\left(b+\frac{\mu}{2}+\frac{1}{2}\right)}{\Gamma\left(\frac{1}{2}\right) \lambda^{2 b+\mu}\left(1+v \lambda^{-2}\right)^{b+\mu / 2+1 / 2}} .
$$

Plugging this in (4.10), we obtain:

$$
J=\frac{\Gamma\left(b+\frac{\mu}{2}+\frac{1}{2}\right)}{\Gamma\left(\frac{1}{2}\right) \Gamma(b+\mu / 2)} \int_{0}^{1} d v v^{b+\mu / 2-1} \frac{(1-v)^{\mu / 2-b}}{\lambda^{2 b+\mu}\left(1+v \lambda^{-2}\right)^{b+\mu / 2+1 / 2}} .
$$

Finally, to derive the desired formula, we use a classical integral representation of ${ }_{2} F_{1}$, together with

$$
{ }_{2} F_{1}(\alpha, \beta, \gamma ;-z)=(1+z)^{\gamma-\alpha-\beta}{ }_{2} F_{1}(\gamma-\alpha, \gamma-\beta, \gamma ;-z)
$$

see formula (9.5.3) in Lebedev [11]. We leave the details to the reader.

\section{References}

[1] Alili, L., Dufresne, D. And Yor, M.: Sur l'identité de Bougerol pour les fonctionnelles exponentielles du mouvement brownien avec drift. In Exponential functionals and principal values related to Brownian motion, 3-14. Bibl. Rev. Mat. Iberoamericana, Rev. Mat. Iberoamericana, Madrid, 1997.

[2] Bertoin, J., Dufresne, D. And Yor, M.: In preparation, December 2012. 
[3] Bertoin, J. And Yor, M.: Retrieving information from subordination. In Prokhorov and Contemporary Probability Theory, 97-106. Springer, 2013.

[4] Bougerol, P.: Exemples de théorèmes locaux sur les groupes résolubles. Ann. Inst. H. Poincaré Sect. B (N.S.) 19 (1983), no. 4, 369-391.

[5] Chaumont, L. And Yor, M.: Exercises in probability. A guided tour from measure theory to random processes, via conditioning. Cambridge Series in Statistical and Probabilistic Mathematics 13, Cambridge University Press, Cambridge, 2003.

[6] Donati-Martin, C., Matsumoto, H. and Yor, M.: On positive and negative moments of the integral of geometric Brownian motions. Stat. Probab. Lett. 49 (2000), no. $1,45-52$.

[7] Dufresne, D.: Laguerre series for Asian and other options. Math. Finance 10 (2000), no.4, 407-428.

[8] Dufresne, D.: The integral of geometric Brownian motion. Adv. Appl. Probab. 33 (2001), no. 1, 223-241.

[9] Dufresne, D. And Yor, M.: A two dimensional extension of Bougerol's identity in law for the exponential of Brownian motion. Working paper no. 222, Centre for Actuarial Studies, University of Melbourne, 2011.

[10] Karatzas, I. And Shreve, S. E.: Brownian motion and stochastic calculus. Graduate Texts in Mathematics 113, Springer-Verlag, New York, 1991.

[11] Lebedev, N. N.: Special functions and their applications. Dover Publications, New York, 1972.

[12] Itô, K. And McKean, H. P.: Diffusions processes and their sample paths. Die Grundlehren der Mathematischen Wissenschaften 125, Academic Press, SpringerVerlag, Berlin-New York, 1965.

[13] McKean, H. P.: Brownian motion and the general diffusion: scale \& clock. In Mathematical finance-Bachelier Congress 2000 (Paris), 75-83. Springer Finance, Springer, Berlin, 2002.

[14] McKean, H. P.: Stochastic integrals. Probability and Mathematical Statistics 5, Academic Press, New York-London, 1969.

[15] Revuz, D. And Yor, M.: Continuous martingales and Brownian motion. Grundlehren der Mathematischen Wissenschaften 293, Springer-Verlag, Berlin, 1999.

[16] Vakeroudis, S.: On hitting times of the winding processes of planar Brownian motion and of Ornstein-Uhlenbeck processes, via Bougerol's identity. Ph.D. Thesis of the University Pierre et Marie Curie, 2011.

[17] Vakeroudis, S.: Bougerol's identity in law and extensions. Probab. Surv., 9 (2012), 411-437.

[18] Yor, M.: Exponential functionals of Brownian motion and related processes. Springer Finance. Springer-Verlag, Berlin, 2001.

[19] Yor, M.: On some exponential functionals of Brownian motion. Adv. in Appl. Probab. 24 (1992), 509-531.

[20] Yor, M. (Editor): Exponential functionals and principal values related to Brownian motion. A collection of research papers. Bibl. Rev. Mat. Iberoamericana, Rev. Mat. Iberoamericana, Madrid, 1997.

Received January 6, 2012. 
JeAn Bertoin: Institut für Mathematik, Universität Zürich, Winterthurerstrasse 190, CH-8057 Zürich, Switzerland.

E-mail: jean.bertoin@math.uzh.ch

Daniel Dufresne: Centre of Actuarial Studies, University of Melbourne, 3010 Victoria, Australia.

E-mail: dufresne@unimelb.edu.au

MARC Yor: Institut Universitaire de France and Laboratoire de Probabilités et Modèles Aléatoires, UPMC, 4 place Jussieu, F-75252 Paris cedex 05, France.

E-mail: deaproba@proba.jussieu.fr 